\title{
Downregulation of long noncoding RNA FENDRR predicts poor prognosis in renal cell carcinoma
}

\author{
WANG HE ${ }^{1,2^{*}}$, GUANGZHENG ZHONG $^{1,2^{*}}$, PEI WANG ${ }^{3 *}$, CHUN JIANG $^{1,2}$, NING JIANG ${ }^{1,2}$ and JIAN HUANG ${ }^{1,2}$ \\ ${ }^{1}$ Department of Urology; ${ }^{2}$ Guangdong Provincial Key Laboratory of Malignant Tumor Epigenetics and Gene Regulation, \\ Sun Yat-Sen Memorial Hospital, Sun Yat-Sen University, Guangzhou, Guangdong 510120; \\ ${ }^{3}$ Department of Emergency Surgery, Shaanxi Provincial People's Hospital, Xi'an, Shaanxi 710068, P.R. China
}

Received April 27, 2017; Accepted July 27, 2018

DOI: $10.3892 / 01.2018 .9624$

\begin{abstract}
Long noncoding RNA FOXF1 adjacent non-coding developmental regulatory RNA (FENDRR) dysregulation associates with multiple types of human cancer. However, the biological functions of FENDRR in renal cell carcinoma are unresolved. Reverse transcription-quantitative polymerase chain reaction (RT-qPCR) was performed to determine the expression level of FENDRR in renal cell carcinoma tissues. An RNA interference assay and ectopic expression experiments were conducted to evaluate the effects of FENDRR on cell proliferation, migration, invasion and colony formation in vitro. RNA immunoprecipitation was conducted to identify proteins associated with FENDRR. It was observed that FENDRR is frequently downregulated in renal cell carcinoma and overexpression of FENDRR attenuated proliferation, migration, invasion and colony growth of renal carcinoma cells. Conversely, knockdown of FENDRR promotes proliferation and invasiveness of renal carcinoma cells. Downregulation of FENDRR associates with poor prognosis of renal cell carcinoma. Mechanistically, it was identified that FENDRR may bind to Polycomb Repressive Complex 2 and lysin methyltransferase $2 \mathrm{~A}$ histone modifying complexes. In summary, FENDRR acts as an tumor suppressor in renal cell carcinoma and may serve as a candidate target for gene therapy.
\end{abstract}

\section{Introduction}

Renal cell carcinoma (RCC) comprises $90 \%$ of all kidney tumors and its incidence is increasing worldwide(1). Worldwide, approximately $30 \%$ of patients with RCC were diagnosed with

Correspondence to: Dr Jian Huang, Department of Urology, Sun Yat-sen Memorial Hospital, Sun Yat-sen University, 107 Yanjiang West Road, Yuexiu, Guangzhou, Guangdong 510120, P.R. China E-mail: urolhj@sina.com

*Contributed equally

Key words: renal cell carcinoma, metastasis, prognosis, long noncoding RNA, RNA-protein interaction metastatic disease, which decreased the 5-year survival rate to only $10 \%$ as reported in $2006(2,3)$. Conventional radical nephrectomy is not able to cure metastatic RCC, which is resistant to radiotherapy and chemotherapy (4). In the past decade, tyrosine kinase inhibitors, also known as targeted therapies, were introduced and demonstrated relatively high rates of response but the 5-year survival rate of patients with metastatic RCC remains low (5). More efficient targets are urgently needed to improve long term survival of patients with advanced RCC.

FOXF1 adjacent non-coding developmental regulatory RNA (FENDRR), a long noncoding RNA lacking an open reading frame of significant length, was first identified as a gene that is specifically expressed in the posterior mesoderm of mouse embryos and is essential for heart and body wall development (6). FENDRR is a conserved transcript of $\sim 3$ kilobases, located on human chromosome 16 (6). Further characterization revealed that it is downregulated in patients with gastric and lung cancer $(7,8)$. Further analysis has demonstrated that histone deacetylation is involved in downregulation of FENDRR and may serve as prognostic factor for human gastric cancer (7).

Although the key functions of FENDRR in embryogenesis and development have been well elucidated $(6,9,10)$, the biological functions of FENDRR and whether aberrant expression of FENDRR occurs in RCC remains to be determined. In the present study, it was identified that FENDRR was frequently downregulated in RCC and other types of human cancer. Overexpression of FENDRR attenuates RCC cells proliferation, migration, invasion and colony formation capabilities, suggesting pivotal functions of FENDRR in RCC progression. Mechanistically, FENDRR physically associates with Polycomb Repressive Complex 2 (PRC2) and lysin methyltransferase 2A (MLL) histone modifying complexes. Importantly, downregulation of FENDRR predicts poor prognosis of RCC.

\section{Materials and methods}

Tissue samples. Samples of fresh frozen RCC cancer tissues, together with normal adjacent tissues ( $3 \mathrm{~cm}$ away from the tumor as in previously published studies) (11), were obtained during radical nephrectomy from Sun Yat-sen Memorial Hospital of Sun Yat-sen University (Guangzhou, China). All samples were 
snap frozen using liquid nitrogen and transferred to $-80^{\circ} \mathrm{C}$ until processed. Tissue samples were collected with written informed consent from patients, and approved by the Ethic Review Committees of Sun-Yat Sen Memorial Hospital. All samples had their diagnoses confirmed pathologically. The nuclear grade was assessed with the Fuhrman grade system (12).

Cell lines and transfection. Human RCC cell lines (769-P, 786-O, ACHN) used in the study were obtained from the American Type Culture Collection (Manassas, VA, USA). 769-P and 786-O cells were cultured in RPMI-1640 medium (Hyclone; GE Healthcare, Logan, UT, USA). ACHN cells were cultured in Dulbecco's modified Eagle's medium (Hyclone; GE Healthcare, Logan, UT, USA). All media were supplemented with $10 \%$ fetal bovine serum (FBS; Hyclone; GE Healthcare) and $1 \%$ penicillin/streptomycin (Gibco; Thermo Fisher Scientific, Inc., Waltham, MA, USA). Cells were cultured in a humidified air atmosphere of $5 \% \mathrm{CO}_{2}$ at $37^{\circ} \mathrm{C}$. Routine tests for mycoplasm infection were negative. Cell transfection was performed using Lipofectamine 2000 or RNAiMAX (Life Technologies; Thermo Fisher Scientific, Inc.) according to the manufacturer's protocol for overexpression or knockdown experiments, respectively. Sequences of siRNAs were as follows: siFENDRR\#1, 5'-CCAGCCAUGUGAUUCCAA ATT-3'; siFENDRR\#2, 5'-GCGAUUGACUGUCUUAUA ATT-3'; siControl, 5'-UUCUCCGAACGUGUCACGUTT-3'.

RNA isolation and reverse transcription-quantitative polymerase chain reaction ( $R T-q P C R$ ). Total RNA from cells was isolated using TRIzol ${ }^{\circledR}$ reagent (Life Technologies; Thermo Fisher Scientific, Inc.) and treated with an RNase-free DNase set (Qiagen China Co., Ltd., Shanghai, China) according to the manufacturer's protocols. RNA electrophoresis on a denaturing agarose gel at 5-6 V/cm was performed to inspect RNA integrity. Intact total RNA run on a denaturing gel has sharp $28 \mathrm{~S}$ and $18 \mathrm{~S}$ rRNA bands, and the 28S rRNA band is approximately twice as intense as the 18S rRNA band. RT was performed using M-MLV reverse transcriptase (Life Technologies; Thermo Fisher Scientific, Inc.) and qPCR was performed with SYBR-Green master mix (Roche Diagnostics, Basel, Switzerland). Relative expression values were calculated using the $2^{-\Delta \Delta \mathrm{Cq}}$ method with GAPDH as a normalizer (13). The primer sequences used were as follows: FENDRR: Forward, 5'-AGAGTGCTTCCACTG CCCTA-3' and reverse, 5'-CCCATTTGCAAAGGCTACAT-3'; GAPDH, forward, 5'-GAAGGTGAAGGTCGGAGTC-3' and reverse, 5'-GAAGATGGTGATGGGATTTC-3'.

Cell proliferation, motility, invasion and colony formation assays. Cell proliferation, motility, invasion and colony formation assays were performed as previously described (14). For the cell proliferation assay, $2 \times 10^{3}$ cells were plated in quadruplicates in 96-well plates and incubated in a humidified air atmosphere of $5 \% \mathrm{CO}_{2}$ at $37^{\circ} \mathrm{C}$. Cell number was calculated using an MTT assay kit (Roche Diagnostics). The cell motility assay was performed using Transwell chambers (BD Falcon; BD Biosciences, San Jose, CA, USA). A total of $1 \times 10^{4}$ cells were plated in Transwell chambers and incubated in complete medium in a humidified air atmosphere of $5 \% \mathrm{CO}_{2}$ at $37^{\circ} \mathrm{C}$ for 8-24h. Cells were stained with $0.1 \%$ crystal violet. For the colony formation assay, 100-500 transfected cells were placed in a fresh six-well plate and maintained for two weeks and colonies were fixed with methanol and stained with $0.1 \%$ crystal violet and counted. An in vitro cell invasion assay was performed using the BD BioCoat Matrigel Invasion Chamber (BD Falcon; BD Biosciences) according to manufacturer's instructions. A total of $1 \times 10^{4}$ cells were plated in BD BioCoat Matrigel Invasion Chambers and incubated in complete medium in a humidified air atmosphere of $5 \% \mathrm{CO}_{2}$ at $37^{\circ} \mathrm{C}$ for $12-36 \mathrm{~h}$. Subsequently, crystal violet on the Transwell membrane was dissolved in $33 \%$ acetic acid solution and measured the absorbance of crystal violet (OD570 nm). Cells were visualized with inverted microscope (magnification, x200; Nikon Corporation, Tokyo, Japan). All experiments were performed in triplicate.

5'-Ethynyl-2'-deoxyuridine (EdU) incorporation assay. 786-O and 769-P cells in S-phase were measured using an EdU assay kit (Guangzhou Ribobio Co., Ltd., Guanzhou, China), according to the manufacturer's protocol. Briefly, cells were incubated with $5 \mu \mathrm{M}$ EdU in culture media supplemented with $10 \% \mathrm{FBS}$ for $2 \mathrm{~h}$ at $37^{\circ} \mathrm{C}$. Then cells were fixed with $4 \%$ formaldehyde for $30 \mathrm{~min}$ at room temperature and treated with $0.5 \%$ Triton $\mathrm{X}-100$ for $20 \mathrm{~min}$ for permeabilization. Subsequent to washing with PBS for 30 seconds at room temperature for three times, the cells were incubated with 1x Apollo ${ }^{\circledR}$ reaction cocktail (Guangzhou RiboBio Co., Ltd.) at $37^{\circ} \mathrm{C}$ for $30 \mathrm{~min}$. Subsequently, the DNA contents were stained with Hoechst $33342(5 \mu \mathrm{g} / \mathrm{ml})$ for $30 \mathrm{~min}$ at $37^{\circ} \mathrm{C}$ and visualized under a fluorescent microscope (magnification, x100; Nikon Corporation, Tokyo, Japan).

RNA immunoprecipitation assay. RNA immunoprecipitation was performed using the EZ-MagnaRIP kit (EMD Millipore, Billerica, MA, USA) according to manufacturer's protocols. Briefly, $5 \times 10^{6}$ cells were harvested and lysed with radioimmunoprecipitation assay lysis buffer with one freeze-thaw cycle. Cell extracts were co-immunoprecipitated with anti-enhancer of zeste 2 polycomb repressive complex 2 (EZH2; 1:500 dilution; cat. no. ab195409, ChIP grade), suppressor of zeste 12 protein homolog (SUZ12; 1:500 dilution; cat. no. ab12073, ChIP grade) and WD repeat domain 5 (WDR5; 1:500 dilution; cat. no. ab56919; ChIP grade; all Abcam, Cambridge, UK), and incubated with agitation overnight at $4^{\circ} \mathrm{C}$. The retrieved RNA was subjected to RT-qPCR analysis. Anti-snRNP70 (1:500 dilution; cat. no. CS203216; EMD Millipore) was used as a technical positive control, and normal mouse Immunoglobulin $\mathrm{G}$ was used as a negative control. For RT-qPCR analysis, U1 splicesome RNA was used as a non-specific control. Experiments were performed in triplicates.

Bioinformatic analysis. Data from The Cancer Genome Atlas (TCGA) were obtained from The Atlas of Noncoding RNAs in Cancer website (http://ibl.mdanderson.org/tanric/_ design/basic/resources.html). Data from the Gene Expression Omnibus (GEO) were downloaded from the GEO website (https://www.ncbi.nlm.nih.gov/geo/).

Statistical analysis. All quantitative data were presented as the mean \pm standard deviation from at least three independent experiments. The differences between two independent groups were analyzed using independent t-tests, and the differences between paired tumor and normal adjacent tissues were 
A

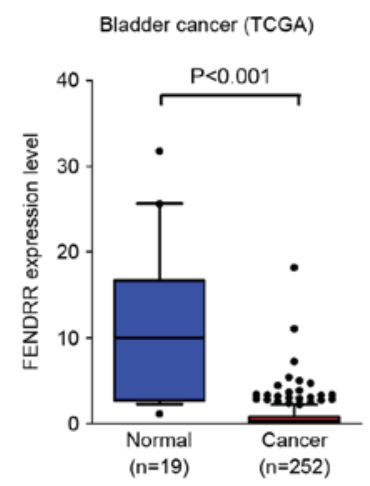

D

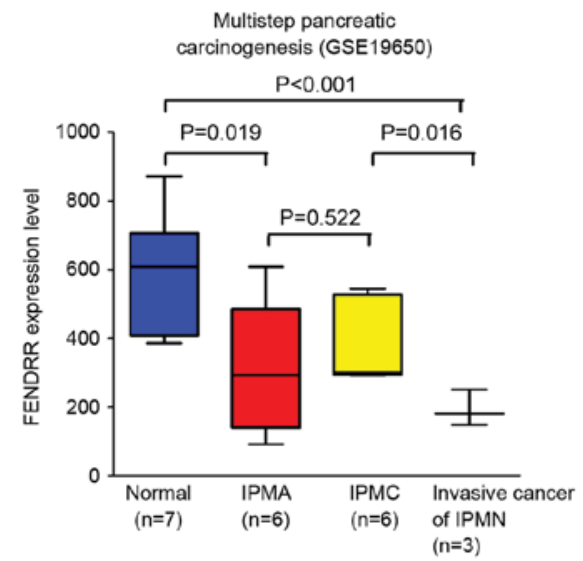

G

Prostate cancer (GSE26910)

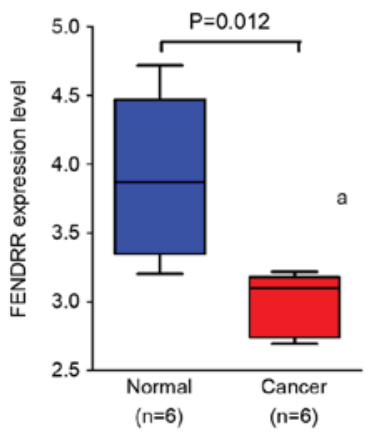

B

Lung cancer (GSE19804)

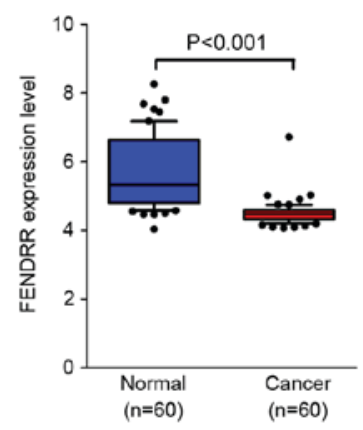

E

Colorectal cancer (GSE32323)

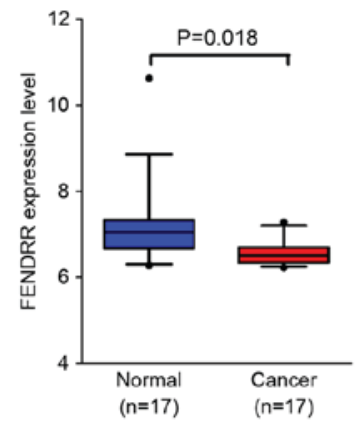

$\mathrm{H}$
C

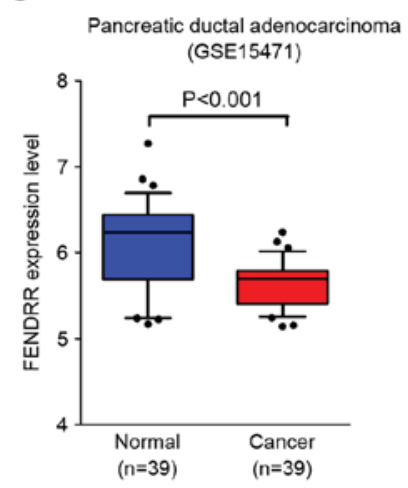

F

Unresectable colorectal cancer primary or metastatic lesions (GSE28702)

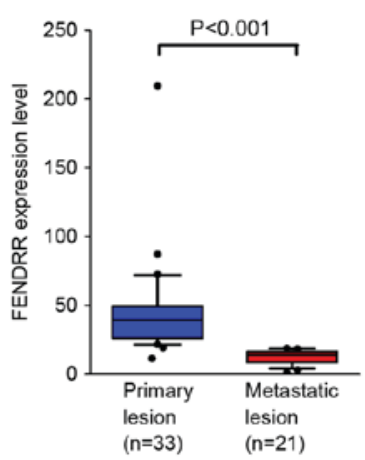

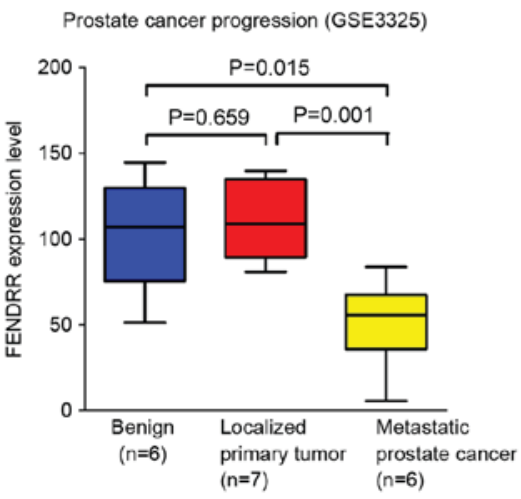

Figure 1. FENDRR is downregulated in multiple human cancers. (A) Analysis of data from TCGA revealed that FENDRR is downregulated in bladder cancer. Data are expressed as box-and-whisker diagrams depicting the smallest observation, 10-90\% percentile, median, and largest observation. Data from GEO demonstrates that FENDRR is downregulated in (B) lung cancer, (C) pancreatic ductal adenocarcinoma, (D) different stages of pancreatic carcinogenesis, (E) colorectal cancer, $(\mathrm{F})$ unresectable colorectal primary or metastatic lesions, $(\mathrm{G})$ prostate cancer and (H) process of prostate cancer progression. Statistical significance was assessed using two-tailed Student's t-tests (A, B, C, E, F and G) and a one-way analysis of variance followed by Dunnett's tests for multiple comparisons (D and H). FENDRR, FOXF1 adjacent non-coding developmental regulatory RNA; TCGA, the cancer genome association; IPMA, intraductal papillary-mucinous adenoma; IPMC, intraductal papillary-mucinous carcinoma; IPMN, intraductal papillary-mucinous neoplasm.

analyzed with paired t-tests. The differences between multiple groups were assessed using one-way analysis of variance followed by Dunnett's multiple-comparison test. The association between FENDRR and clinical characteristics was analyzed using Pearson Chi-square test. All tests performed are two sided. Statistical tests were performed using SPSS software (version 10.0; SPSS, Inc., Chicago, IL, USA). P<0.05 was considered to indicate a statistically significant difference.

\section{Results}

FENDRR is downregulated in multiple human cancers. To explore the biological roles of FENDRR in human cancer, publically available databases were searched. As presented in Fig. 1, FENDRR is downregulated in multiple types of human cancer, including bladder and lung cancer, and pancreatic ductal adenocarcinoma. Interestingly, in 
Table I. Characteristics of renal cell carcinoma patients.

\section{FENDRR}

\begin{tabular}{|c|c|c|c|c|c|}
\hline Characteristic & Patient frequency $(\%)$ & Low & High & Pearson $\chi^{2}$ & P-value \\
\hline Total & 56 & $41(73.2 \%)$ & $15(26.8 \%)$ & & \\
\hline \multicolumn{6}{|l|}{ Sex } \\
\hline Male & $39(69.6)$ & 30 & 9 & 0.386 & 0.535 \\
\hline Female & $17(30.4)$ & 11 & 6 & & \\
\hline \multicolumn{6}{|l|}{ Age, years } \\
\hline$<60$ & $32(57.1)$ & 25 & 7 & 0.918 & 0.338 \\
\hline$\geq 60$ & $24(42.9)$ & 16 & 8 & & \\
\hline \multicolumn{6}{|l|}{ Tumor stage } \\
\hline $\mathrm{T} 1$ & $38(67.9)$ & 26 & 12 & 0.730 & 0.39 \\
\hline $\mathrm{T} 2-4$ & $18(32.1)$ & 15 & 3 & & \\
\hline \multicolumn{6}{|l|}{ Fuhrman grade } \\
\hline $1-2$ & $34(60.7)$ & 21 & 13 & 5.785 & 0.016 \\
\hline $3-4$ & $22(39.3)$ & 20 & 2 & & \\
\hline
\end{tabular}

FENDRR, FOXF1 adjacent non-coding developmental regulatory RNA.
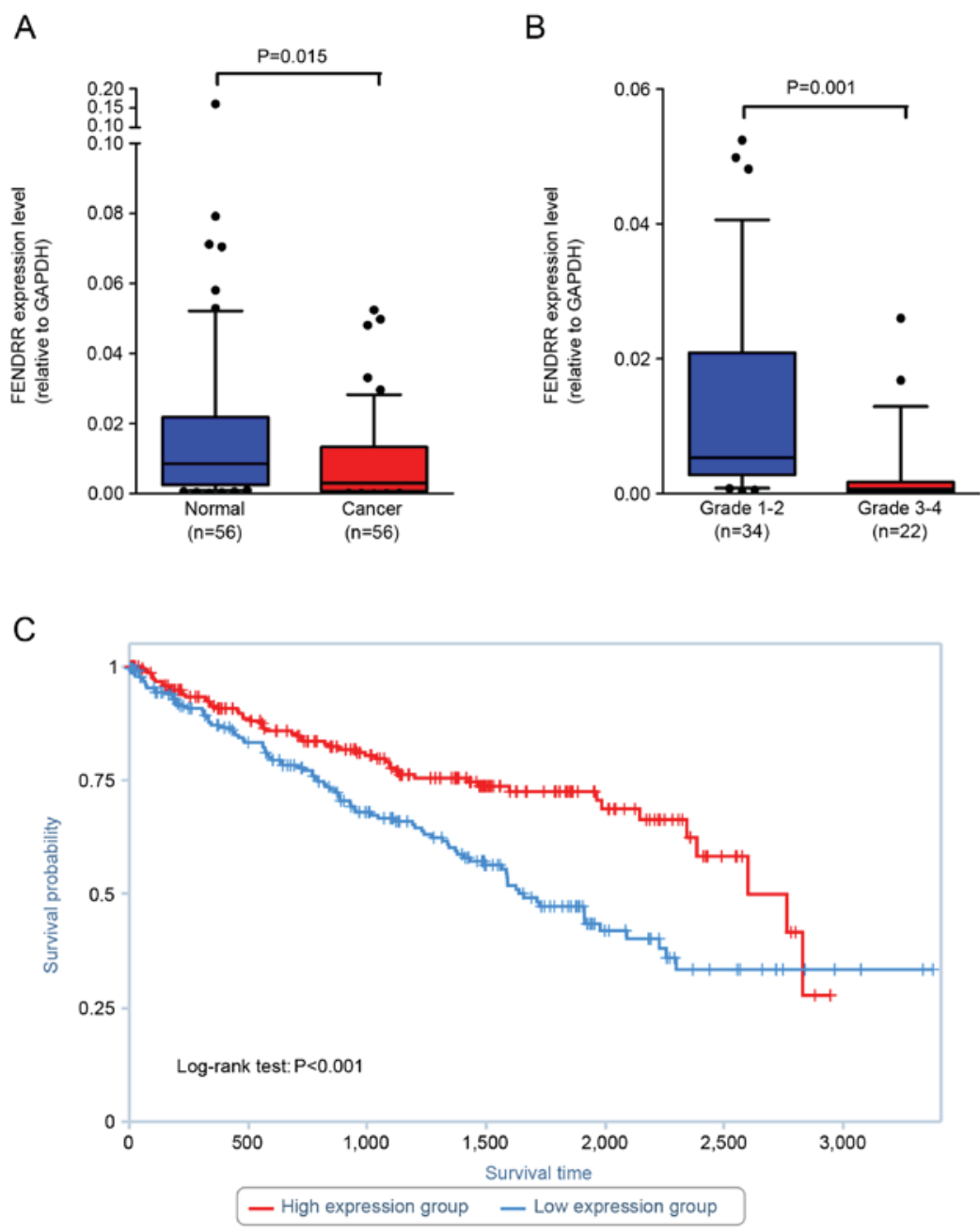

Figure 2. FENDRR is downregulated in renal clear cell carcinoma and correlated with poor prognosis. Box-and-whisker diagrams presenting the expression level of FENDRR in (A) normal adjacent tissues and renal cell carcinoma tissues and (B) different Fuhrman grade RCC tissues. (C) The cancer genome atlas data presents that a lower expression level of FENDRR associates with poor prognosis of patients with renal clear cell carcinoma. FENDRR, FOXF1 adjacent non-coding developmental regulatory RNA. 
A

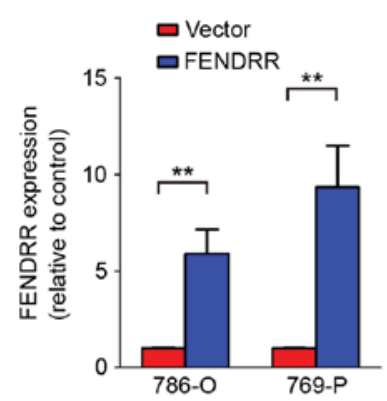

D

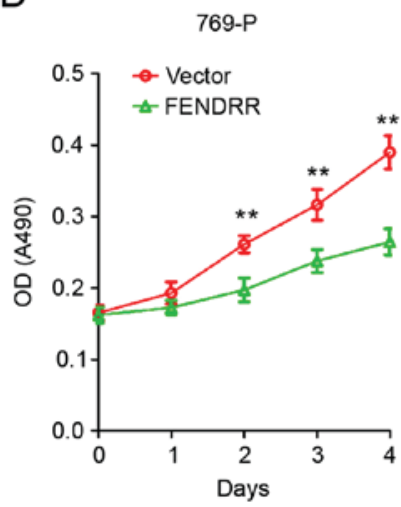

B

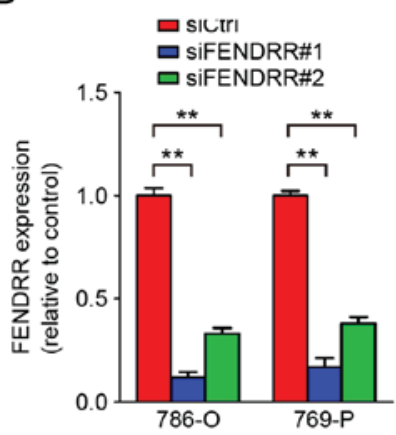

E

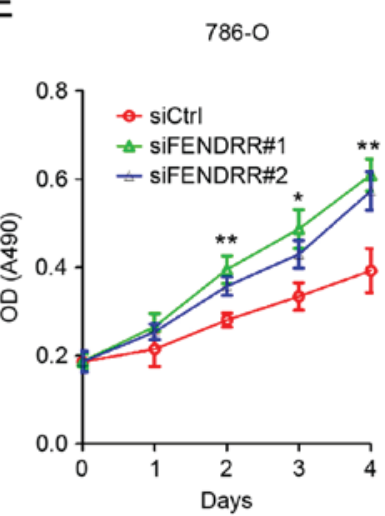

C

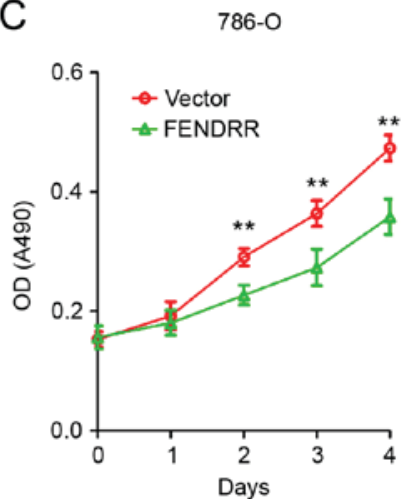

F

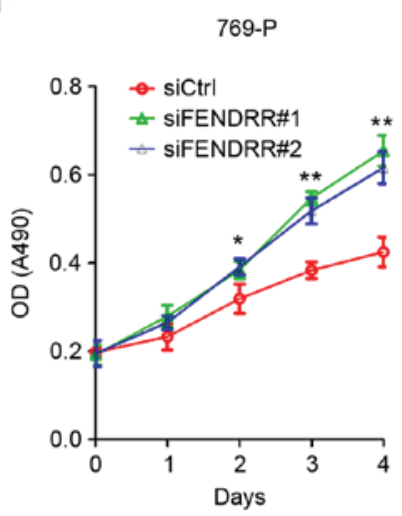

G $786-0$

DAPI

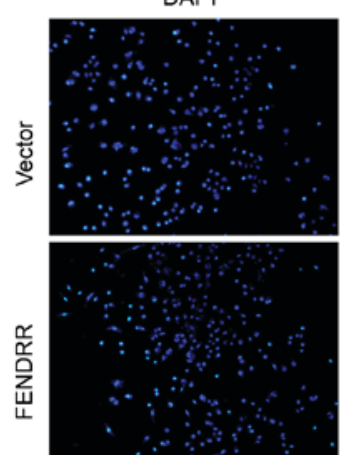

EdU

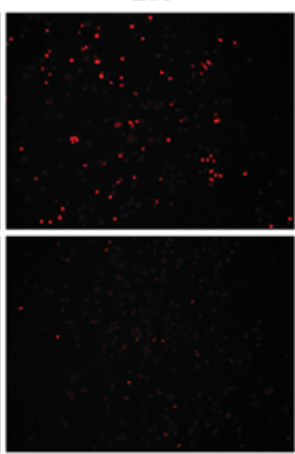

DAPI

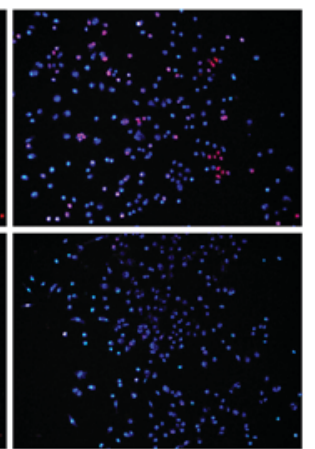

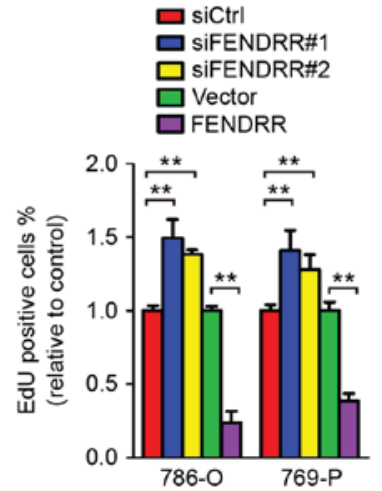

Figure 3. FENDRR inhibits proliferation of RCC cells. (A) Efficiencies in ectopic expression experiments as determined by qRT-PCR. (B) Efficiencies in knockdown experiments as determined by qRT-PCR. (C) MTT assay demonstrating the effects FENDRR overexpression on proliferation of 786-O cell line. (D) MTT assay demonstrating the effects of FENDRR overexpression on proliferation of 769-P cell line. (E) MTT assay demonstrating the effects of siRNAs targeting FENDRR on proliferation of 786-O cell line. (F) MTT assay demonstrating the effects of siRNAs targeting FENDRR on proliferation of 769-P cell line. (G) EdU assay presenting the effects of FENDRR on the proportion of RCC cells in S phase. Blue color represents the nucleus and red color indicates $\mathrm{S}$ phase cells (EdU positive). Statistical significance was assessed using two-tailed Student's t-tests (A, C and D) and a one-way analysis of variance followed by Dunnett's tests for multiple comparisons (B, E, F and G). "P $<0.05$ and ${ }^{* *} \mathrm{P}<0.01$. FENDRR, FOXF1 adjacent non-coding developmental regulatory RNA; RCC, renal cell carcinoma; EdU, 5'-Ethynyl-2'-deoxyuridine.

the observation of multistep pancreatic carcinogenesis, the expression levels of FENDRR were downregulated in intraductal papillary mucinous adenoma, intraductal papillary mucinous carcinoma and invasive cancer of intraductal papillary mucinous neoplasm. More importantly, the expression levels of FENDRR were downregulated in the metastatic site compared with the primary tumor in colorectal cancer and prostate cancer.

Collectively, these data demonstrated that FENDRR serves important functions in carcinogenesis and cancer metastasis in multiple types of human cancer.
FENDRR is downregulated in renal cell carcinoma and associates with poor prognosis of RCC. In order to determine whether dysregulation of FENDRR expression indeed occurs in renal cell carcinoma, the RNA level of FENDRR was quantified in renal cell carcinoma tissues and paired normal adjacent cortex tissues using RT-qPCR. Fold changes of $<1.0$ were designated as downregulated in cancer tissues compared with normal adjacent tissues. The results revealed that FENDRR was downregulated in $73.2 \%$ of renal cell carcinoma tissues (Table I; Fig. 2A). Statistical analysis revealed that lower FENDRR expression was associated with Fuhrman 

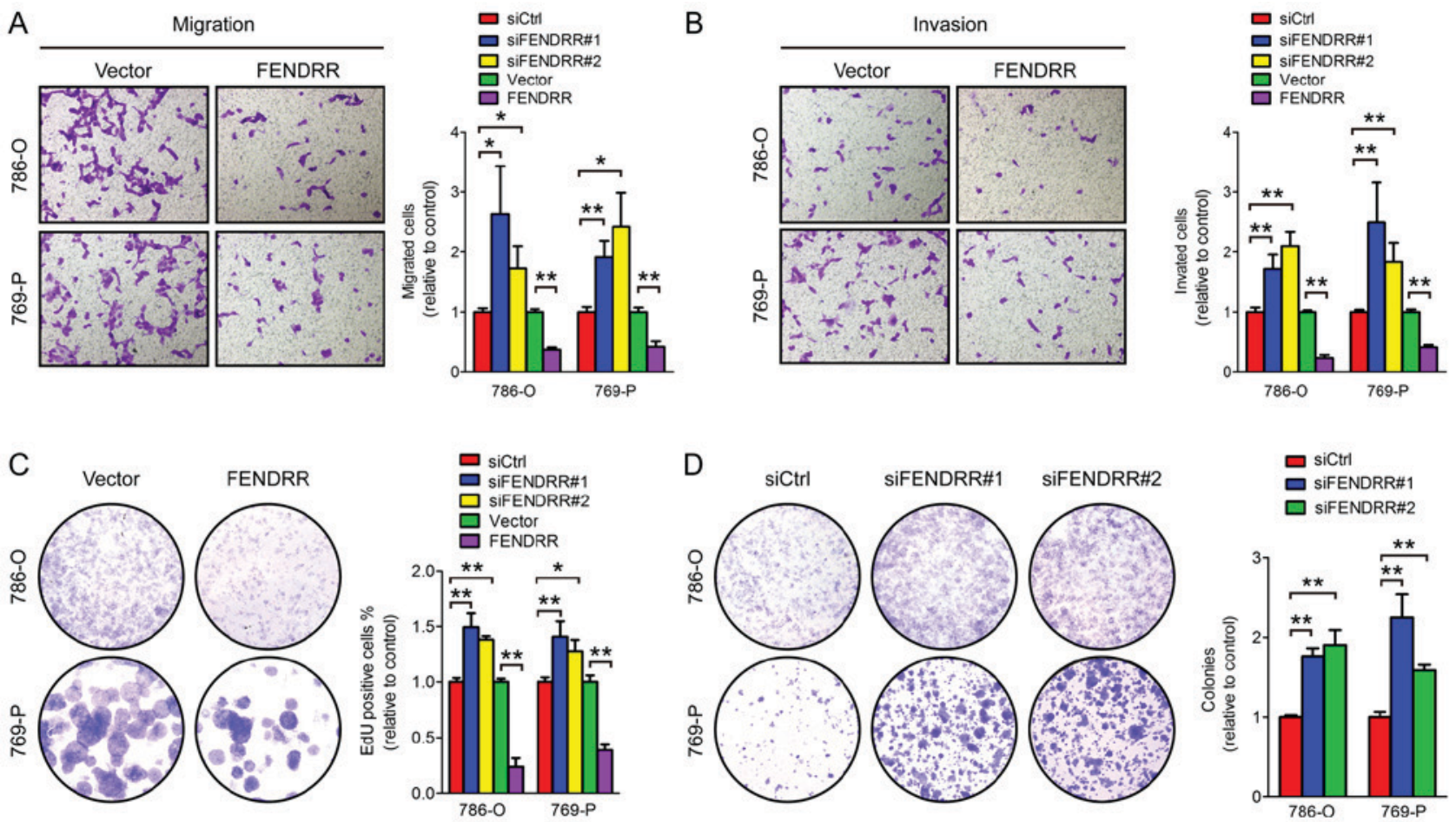

Figure 4. FENDRR inhibits migration and invasion of renal carcinoma cells. (A) Representative images of cell migration following overexpression of FENDRR. Histograms summarize the effects of overexpression or knockdown of FENDRR on cell migration in 786-O and 769-P cell lines in at least three independent experiments. (B) Representative images of cell invasion following overexpression of FENDRR. Histograms summarize the effects of overexpression or knockdown of FENDRR on cell invasion in 786-O and 769-P cell lines in at least three independent experiments. (C) Representative images of colonies formation assay and the histological analysis of the number of colonies following overexpression of FENDRR. (D) Representative images of colonies formation assay and the histological analysis of the number of colonies following knockdown of FENDRR. Statistical significance was assessed using one-way analysis of variance followed by Dunnett's tests for multiple comparisons. FENDRR, FOXF1 adjacent non-coding developmental regulatory RNA. "P $<0.05$ and ${ }^{* *} \mathrm{P}<0.01$.

grade (Fig. 2B). As a result of the limited number of cases and the follow up period, TCGA database was searched to identify whether FENDRR expression associates with the prognosis of patients with renal cell carcinoma. The results demonstrated that lower FENDRR expression associates with shorter overall survival ( $\mathrm{P}=0.00025$; Fig. 2C).

FENDRR downregulation results in accelerated cell proliferation of RCC cells. Unlimited proliferation ability comprises one of the most important characteristics of malignant tumor. Reports have identified that downregulation of FENDRR promotes tumor growth and associates with poor prognosis in gastric cancer (7). To further determine the role of FENDRR in regulating RCC cell proliferation, FENDRR was overexpressed using expression vectors or depleted in RCC cell lines using siRNAs. As presented in Fig. 3A-D, knockdown of endogenous FENDRR accelerated cell proliferation in 786-O and 769-P RCC cells, compared with the non-specific control siRNA that shared no homology with the human genome. Conversely, overexpression of FENDRR significantly inhibited proliferation of RCC cells (Fig. 3E-F). These data suggested a function of FENDRR in regulating RCC cell proliferation.

To further validate the role of FENDRR in cell cycle modulation, an EdU incorporation assay was performed. EdU is a nucleotide analog of thymidine and is incorporated into DNA during DNA synthesis. Thus EdU positive cells indicate the cell is in $\mathrm{S}$ phase. Markedly increased EdU positive cells were observed following FENDRR depletion in 786-O and
769-P RCC cells (Fig. 3G), and overexpression of FENDRR decreased the proportion of cells in $\mathrm{S}$ phase, further indicating that FENDRR expression inhibited cell proliferation of RCC cells.

FENDRR overexpression inhibits the migration, invasion and colony formation abilities of RCC cells. The ability to invade the basement membrane and migrate from the primary site to a distant site is critical for malignant cancer cells to form metastatic lesions. Cell migration ability was evaluated using Transwell chambers and cell invasion was assessed using a Matrigel invasion assay which mimics the process of tumor cells invading the basement membrane. As presented in Fig. 4A-B, FENDRR knockdown promoted, while FENDRR overexpression inhibited, cell migration and invasion of RCC cells, suggesting that FENDRR serves a regulatory function in RCC cell migration and invasion.

Colony formation ability demonstrates the cell-population dependent proliferation capacity of cancer cells. Knockdown of endogenous FENDRR markedly promoted, and FENDRR overexpression inhibited, colony formation both in 786-O and 769-P cells (Fig. 4C-D). Together, these results indicate the critical function of FENDRR in the process of metastasis of renal cell carcinoma.

Potential targets of FENDRR in RCC. To identify potential target genes of FENDRR involved in the metastasis of RCC, an analysis of FENDRR and mRNA expression was performed using TCGA database. As presented in Fig. 5, 
A

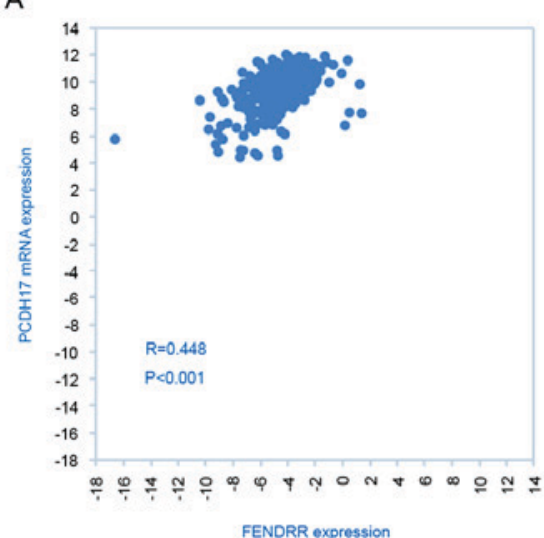

D

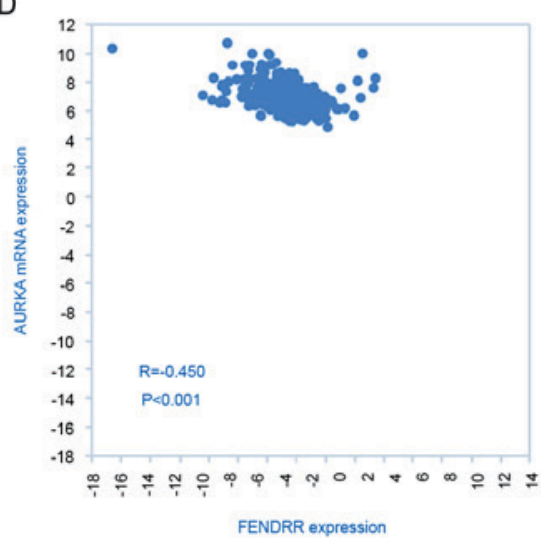

B

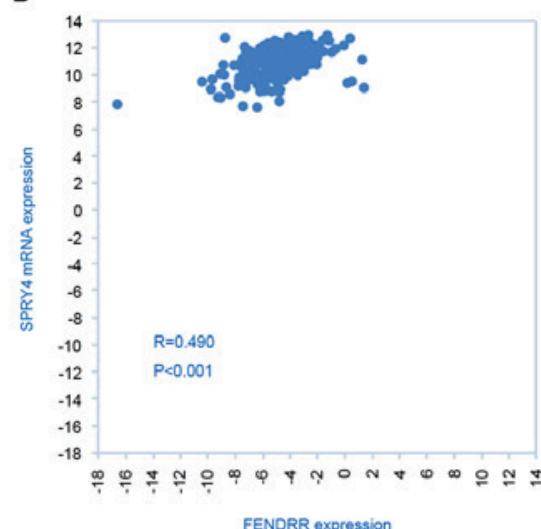

E

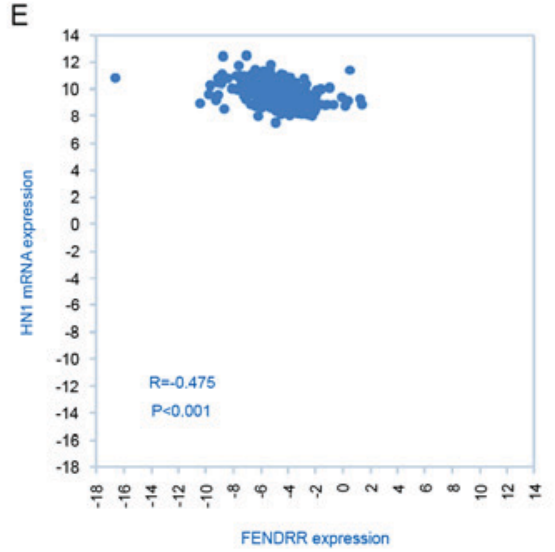

C

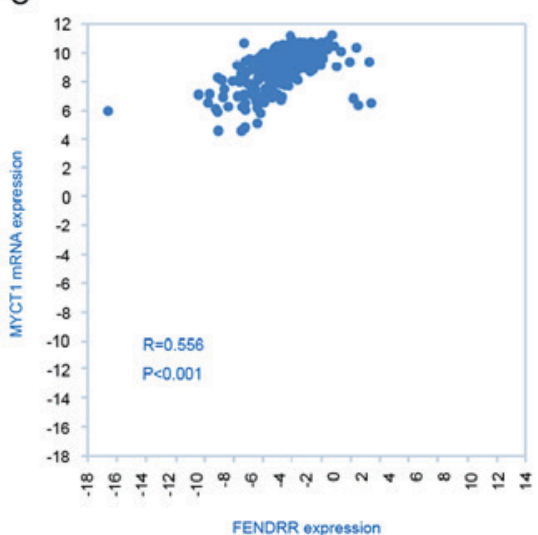

F

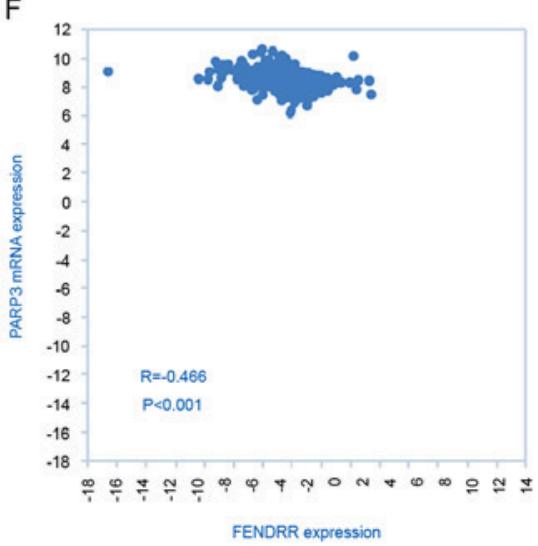

Figure 5. Potential down-stream target genes of FENDRR. (A) Analysis of TCGA database (TCGA) revealed positive correlation of FENDRR expression PCDH17. (B) FENDRR expression positively correlates with SPRY4 in TCGA database. (C) FENDRR expression positively correlates with MYCT1. (D) FENDRR expression negatively correlates with AURKA. (E) FENDRR expression negatively correlates with HN1. (F) FENDRR expression negatively correlates with AURKA. PARP3. Statistical significance was assessed using Pearson's correlation analysis. FENDRR, FOXF1 adjacent non-coding developmental regulatory RNA; PCDH17, proctocadherin 17; SPRY4, sprouty RTK signaling antagonist 4; MYCT1, MYC target 1; AURKA, aurora kinase A; PARP3, poly(ADP-ribose) polymerase family member 3; TCGA, The Cancer Genome Atlas.

a positive association was identified between FENDRR expression and tumor suppressors protocadherin 17, sprouty RTK signaling antagonist 4, MYC target 1 and a negative association was identified between FENDRR and oncogene aurora kinase A, jupiter microtubule associated homolog 1 and poly(ADP-ribose) polymerase family member 3, suggesting that FENDRR may serve as a tumor suppressor by activating important tumor suppressor genes and inhibiting expression of oncogenes.

FENDRR physically associates with PRC2 complex and MLL complex. To further elucidate how FENDRR regulates down-stream target genes, we next performed RNA immunoprecipitation to identify FENDRR interacting proteins. Our RIP assay confirmed that FENDRR physically associates with EZH2, SUZ12 and WDR5 in RCC cells (Fig. 6), suggesting that FENDRR may exert its function at least partially by interacting with PRC2 complex and MLL complex.

\section{Discussion}

FENDRR has been suggested to serve important functions in vertebral development and is downregulated in numerous types of cancer (7-9,15). A low level of FENDRR expression promotes local invasion and lymphatic metastasis in gastric cancer, which are key steps for cancer progression (7). To interpret how this transcript exerts its biological function, potential mechanisms were proposed, including controlling epigenetic modifications of promoters of target genes critical for embryonic development (6), and promoting cancer invasion through regulating expression of matrix metalloproteinase (MMP)2/MMP9 (7). In the present study, it was identified that FENDRR downregulation occurs in the process of carcinogenesis and cancer metastasis through searching a publicly available database. Though a tumor suppressing role was identified in multiple types of human cancer, functional characterization of FENDRR in RCC remains undocumented. Consistent with observations in other cancers, the present data revealed that FENDRR downregulation is very common in RCC and associates with poor prognosis of patients with RCC. Importantly, overexpression of FENDRR significantly inhibited cell proliferation and invasion, pointing to the possibility of FENDRR as a therapeutic target for cancer treatment.

Dysregulation of chromatin modifications including $\mathrm{H} 3 \mathrm{~K} 4$ and H3K27 methylation is implicated in the carcinogenesis of RCC (16). The MLL complex is responsible for 
A
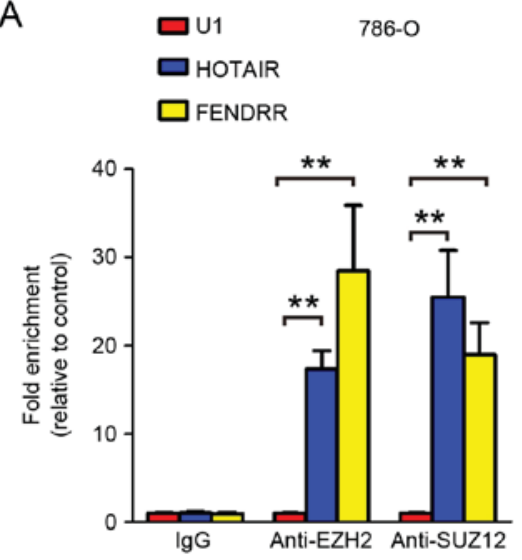

C
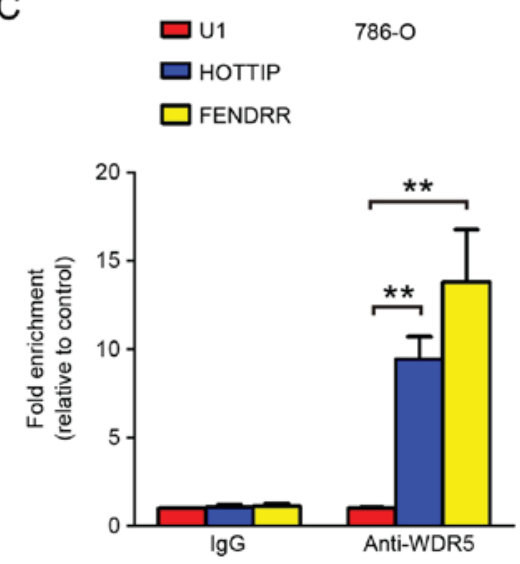

B
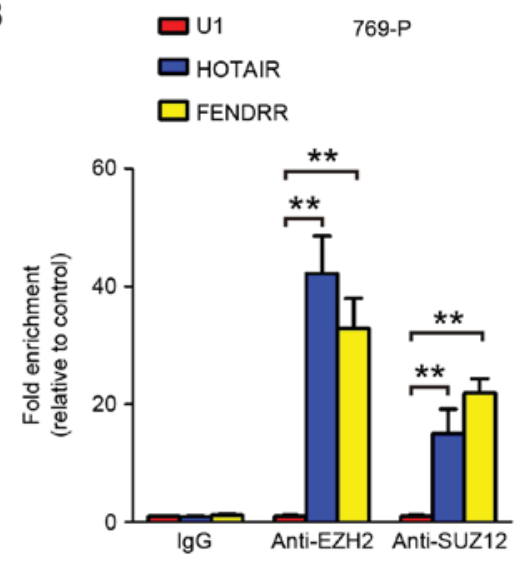

D

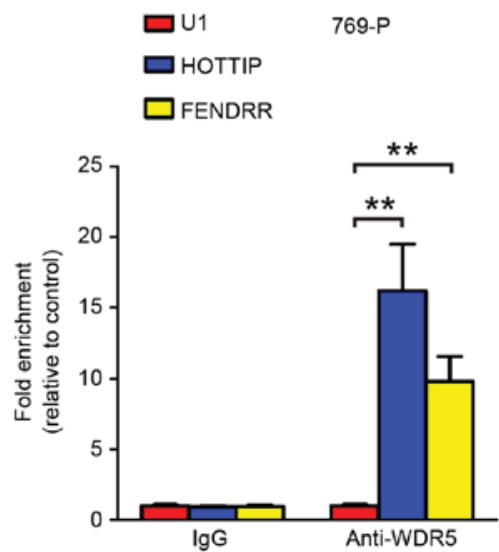

Figure 6. FENDRR physically associates with enhancer of zeste 2 polycomb repressive complex 2 and WDR5 protein. Histograms presenting an RNA immunoprecipitation assay using antibodies to EZH2 and SUZ12 as baits to co-immunoprecipitate RNAs that bind to these proteins in (A) 786-O and (B) 769-P cells. The achieved RNAs were subjected to reverse transcription-quantitative polymerase chain reaction to assess the quantity of FENDRR. A radioimmunoprecipitation assay was performed using an antibody against WDR5 in (C) 786-O and (D) 769-P cells. Statistical significance was assessed using one-way analysis of variance followed by Dunnett's tests for multiple comparisons. FENDRR, FOXF1 adjacent non-coding developmental regulatory RNA; WDR5, WD repeat domain 5; EZH2, enhancer of zeste 2 polycomb repressive complex 2; SUZ12, suppressor of zeste 12 protein homolog; HOTAIR, HOX transcript antisense RNA; HOTTIP, HOXA transcript at the distal tip; Ig, immunoglobulin. ${ }^{* *} \mathrm{P}<0.01$.

H3K4 methylation (17) and polycomb group proteins (PRC1 and PRC2) are epigenetic silencers implicated in cancer development (18). EZH2, a core component of PRC2, directly controls the histone $\mathrm{H} 3$ lysine 27 trimethylation and interacts with DNA methyltransferases promoting DNA methylation, and EZH2 is critical for these processes $(19,20)$ and predicts cancer specific survival of RCC (16). Studies have uncovered that numerous long intergenic noncoding RNAs (lincRNAs) associate with epigenetic modifying complexes, and $>20 \%$ of lincRNAs interact with PRC2 complex (21). In the present study, the gathered data suggested that FENDRR is able to physically associate with WDR5, EZH2 and SUZ12 in renal carcinoma cells. It is of note that certain studies provide evidence that EZH2 promotes the progression of RCC by modulating vascular endothelial growth factor and E-cadherin expression $(22,23)$. However, a more precise mechanism detailing how FENDRR affects the targeting specificity of PRC2 complex and MLL complex to promote carcinogenesis and cancer metastasis remains unresolved and warrants further investigation.

It is a common phenomenon that genes associated with developmental process also serve important functions in cancer progression. Recent studies have demonstrated that long noncoding RNAs serve important functions in the process of limb development and lymph node metastasis of multiple types of cancer (HOXA transcript at the distal tip) $(24,25)$, skeletal development and cancer metastasis (HOX transcript antisense RNA) (24), muscle differentiation and cancer metastasis (H19) (24,26). Specifically, FENDRR is dispensable for lung and heart development, and others and the present experiments suggested that FENDRR binds to PRC2 and MLL complexes $(6,9)$. Markedly, the present study identified that FENDRR is not only critical for RCC but also serves a function in the development of multiple cancers. However, the precise molecular mechanisms by which FENDRR affects cellular phenotypic alterations, including cell proliferation and migration, is largely unexplored, which requires further investigation in future studies.

To conclude, the present study reports that the long noncoding RNA FENDRR acts as a tumor suppressor in RCC. It was identified that FENDRR is frequently downregulated in cancerous tissues of RCC, promoting cell migration and invasion. Furthermore, overexpression of FENDRR decelerates cell migration, invasion and colony formation of RCC cells. 
These findings suggest that targeting FENDRR may help controlling RCC progression and metastasis.

\section{Acknowledgements}

The authors would like to thank Dr. Changhao Chen (Sun Yat-sen University) for helpful discussion of the article. Mr. Wang He is a recipient of Yat-sen Scholarship for Young Scientists.

\section{Funding}

The present study was funded by the National Natural Science Foundation of China (grant nos. 81472381, 81572514, U1301221, 81472384, 81402106, 81372729, $81272808,81172431)$, the Science and Technology Program of Guangzhou (grant nos. 201707010116, 201604020156, 201604020177), the National Natural Science Foundation of Guangdong (grant nos. 2016A030313321, 2015A030311011, 2015A030310122, S2013020012671, 07117336, 10151008901000024), the Three Big Construction funds of Sun Yat-sen University, the Specialized Research Fund for the Doctoral Program of Higher Education (grant no. 20130171110073), the Fundamental Research Funds for the Central Universities, the Guangdong Province Higher Vocational Colleges \& Schools Pearl River Scholar Funded Scheme, the Elite Young Scholars Program of Sun Yat-Sen Memorial Hospital (grant no. J201401), the National Clinical Key Specialty Construction Project for Department of Urology and Department of Oncology, the Key Laboratory of Malignant Tumor Gene Regulation and Target Therapy of Guangdong Higher Education Institutes, Sun-Yat-Sen University (grant no. KLB09001) and the Key Laboratory of Malignant Tumor Molecular Mechanism and Translational Medicine of Guangzhou Bureau of Science and Information Technology [grant no. (2013)163].

\section{Availability of data and materials}

The datasets generated and analyzed during the current study are available in the TCGA repository, http://ibl.mdanderson. org/tanric/_design/basic/index.html.

\section{Authors' contributions}

WH and JH participated in the study design. GZ, PW and NJ carried out in vitro cell experiments and data analysis. PW and CJ performed the PCR experiments and data analysis. WH conceived the study and wrote the manuscript. All the authors read and approved the final manuscript.

\section{Ethics approval and consent to participate}

Tissue samples were collected with written informed consent from patients, and approved by the Ethics Review Committee of Sun Yat-sen Memorial Hospital.

\section{Patient consent for publication}

All samples were collected with written informed consent from patients. No identifying information, including names, initials, date of birth or hospital numbers, images or statements were included in the manuscript.

\section{Competing interests}

The authors declare that they have no competing interests.

\section{References}

1. Jonasch E, Gao J and Rathmell WK: Renal cell carcinoma. BMJ 349: g4797, 2014.

2. Hollingsworth JM, Miller DC, Daignault S and Hollenbeck BK: Five-year survival after surgical treatment for kidney cancer: A population-based competing risk analysis. Cancer 109: 1763-1768, 2007.

3. Gupta K, Miller JD, Li JZ, Russell MW and Charbonneau C: Epidemiologic and socioeconomic burden of metastatic renal cell carcinoma (mRCC): A literature review. Cancer Treat Rev 34: 193-205, 2008.

4. Mourad WF, Dutcher J and Ennis RD: State-of-the-art management of renal cell carcinoma. Am J Clin Oncol 37: 498-505, 2014

5. Escudier B, Eisen T, Stadler WM, Szczylik C, Oudard S, Staehler M, Negrier S, Chevreau C, Desai AA, Rolland F, et al: Sorafenib for treatment of renal cell carcinoma: Final efficacy and safety results of the phase III treatment approaches in renal cancer global evaluation trial. J Clin Oncol 27: 3312-3318, 2009.

6. Grote P, Wittler L, Hendrix D, Koch F, Währisch S, Beisaw A, Macura K, Bläss G, Kellis M, Werber M and Herrmann BG: The tissue-specific lncRNA Fendrr is an essential regulator of heart and body wall development in the mouse. Dev Cell 24: 206-214, 2013.

7. Xu TP, Huang MD, Xia R, Liu XX, Sun M, Yin L, Chen WM, Han L, Zhang EB, Kong R, De W, et al: Decreased expression of the long non-coding RNA FENDRR is associated with poor prognosis in gastric cancer and FENDRR regulates gastric cancer cell metastasis by affecting fibronectin 1 expression. J Hematol Oncol 7: 63, 2014.

8. Chen R, Li WX, Sun Y, Duan Y,Li Q, Zhang AX, Hu JL, Wang YM and Gao YD: Comprehensive analysis of lncRNA and mRNA expression profiles in lung cancer. Clin Lab 63: 313-320, 2017.

9. Sauvageau M, Goff LA, Lodato S, Bonev B, Groff AF, Gerhardinger C, Sanchez-Gomez DB, Hacisuleyman E, Li E, Spence M, et al: Multiple knockout mouse models reveal lincRNAs are required for life and brain development. Elife 2: $\mathrm{e} 01749,2013$.

10. Grote P and Herrmann BG: The long non-coding RNA Fendrr links epigenetic control mechanisms to gene regulatory networks in mammalian embryogenesis. RNA Biol 10: 1579-1585, 2013.

11. Wang $\mathrm{H}$ and Men CP: Correlation of increased expression of MicroRNA-155 in bladder cancer and prognosis. Lab Med 46: 118-122, 2015.

12. Fuhrman SA, Lasky LC and Limas C: Prognostic significance of morphologic parameters in renal cell carcinoma. Am J Surg Pathol 6: 655-663, 1982.

13. Livak KJ and Schmittgen TD: Analysis of relative gene expression data using real-time quantitative PCR and the 2(-Delta Delta C(T)) Method. Methods 25: 402-408, 2001.

14. He W, Cai Q, Sun F, Zhong G, Wang P, Liu H, Luo J, Yu H, Huang $J$ and Lin T: linc-UBC1 physically associates with polycomb repressive complex 2 (PRC2) and acts as a negative prognostic factor for lymph node metastasis and survival in bladder cancer. Biochim Biophy Acta 1832: 1528-1537, 2013.

15. Li Q, Wu C, Song G, Zhang H, Shan B, Duan Y and Wang Y: Genome-wide analysis of long noncoding RNA expression profiles in human Xuanwei lung cancer. Clin Lab 61: 1515-1523, 2015.

16. Vieira-Coimbra M, Henrique R and Jeronimo C: New insights on chromatin modifiers and histone post-translational modifications in renal cell tumours. Eur J Clin Invest 45 (Suppl) 1: S16-S24, 2015.

17. Schuettengruber B, Chourrout D, Vervoort M, Leblanc B and Cavalli G: Genome regulation by polycomb and trithorax proteins. Cell 128: 735-745, 2007.

18. Schlesinger Y, Straussman R, Keshet I, Farkash S, Hecht M, Zimmerman J, Eden E, Yakhini Z, Ben-Shushan E, Reubinoff BE, et al: Polycomb-mediated methylation on Lys27 of histone $\mathrm{H} 3$ pre-marks genes for de novo methylation in cancer. Nat Genetics 39: 232-236, 2007. 
19. Wang Y, Chen Y, Geng H, Qi C, Liu Y and Yue D: Overexpression of YB1 and EZH2 are associated with cancer metastasis and poor prognosis in renal cell carcinomas. Tumour Biol 36: 7159-7166, 2015.

20. Ning X, Shi Z, Liu X, Zhang A, Han L, Jiang K, Kang C and Zhang Q: DNMT1 and EZH2 mediated methylation silences the microRNA-200b/a/429 gene and promotes tumor progression. Cancer Lett 359: 198-205, 2015.

21. Khalil AM, Guttman M, Huarte M, Garber M, Raj A, Rivea Morales D, Thomas K, Presser A, Bernstein BE, van Oudenaarden A, et al: Many human large intergenic noncoding RNAs associate with chromatin-modifying complexes and affect gene expression. Proc Natl Acad Sci USA 106: 11667-11672, 2009.

22. Liu L, Xu Z, Zhong L, Wang H, Jiang S, Long Q, Xu J and Guo J: EZH2 promotes tumor cell migration and invasion via epigenetic repression of E-cadherin in renal cell carcinoma. BJU Int 117: 351-362, 2016

23. Xu ZQ, Zhang L, Gao BS, Wan YG, Zhang XH, Chen B, Wang YT, Sun N and Fu YW: EZH2 promotes tumor progression by increasing VEGF expression in clear cell renal cell carcinoma. Clin Transl Oncol 17: 41-49, 2015.
24. Schmitz SU, Grote P and Herrmann BG: Mechanisms of long noncoding RNA function in development and disease. Cell Mol Life Sci 73: 2491-2509, 2016.

25. Chen Z, He A, Wang D, Liu Y and Huang W: Long noncoding RNA HOTTIP as a novel predictor of lymph node metastasis and survival in human cancer: A systematic review and meta-analysis. Oncotarget 8: 14126-14132, 2017.

26. Jing W, Zhu M, Zhang XW, Pan ZY, Gao SS, Zhou H, Qiu SL, Liang CZ and Tu JC: The significance of long noncoding RNA H19 in lredicting progression and metastasis of cancers: A Meta-Analysis. Biomed Res Int 2016: 5902678, 2016.

This work is licensed under a Creative Commons Attribution-NonCommercial-NoDerivatives 4.0 International (CC BY-NC-ND 4.0) License. 